\title{
USO DAS MADEIRAS DE SERINGUEIRA, PINUS E CIPRESTE NA FABRICAÇÃO DE CHAPAS OSB
}

\author{
Esmeralda Yoshico Arakaki Okino ${ }^{1}$, Divino Eterno Teixeira ${ }^{2}$, Mário Rabelo de Souza ${ }^{3}$, \\ Marcos Antonio Eduardo Santana ${ }^{4}$, Camila Bezerra Gomes da Silva ${ }^{5}$, \\ Raoni Blum Tomaz ${ }^{6}$, Maria Eliete de Sousa ${ }^{7}$ \\ ${ }^{1}$ Eng $^{a}$. Química, M.Sc., IBAMA, Brasília, DF, Brasil - esmeralda.okino@ florestal.gov.br \\ ${ }^{2}$ Eng. Florestal, Ph.D., IBAMA, Brasília, DF, Brasil - divino.teixeira@ florestal.gov.br \\ ${ }^{3}$ Físico, Ph.D., IBAMA, Brasília, DF, Brasil - mario.souza@ florestal.gov.br \\ ${ }^{4}$ Químico, Ph.D., IBAMA, Brasília, DF, Brasil - marcos.santana@ florestal.gov.br \\ ${ }^{5}$ Eng $^{\text {a }}$. Florestal, Brasília, DF, Brasil - kamira.yoruno@gmail.com.br \\ ${ }^{6}$ Eng. Florestal, Brasília, DF, Brasil - raonibt@terra.com.br \\ ${ }^{7}$ Enga . Química, Brasília, DF, Brasil - maria-eliete.sousa@florestal.gov.br
}

Recebido para publicação: 07/11/2007 - Aceito para publicação: 03/09/2008

\begin{abstract}
Resumo
Este trabalho avaliou as propriedades físicas e mecânicas de chapas de OSB usando madeiras de pinus, de cipreste e da mistura equitativa de dois clones de seringueira. Foram confeccionadas chapas de três camadas, na proporção face/miolo/face, de $1: 2: 1$, massa específica de $0,75 \mathrm{~g} / \mathrm{cm}^{3}$ e resinas ureia-formaldeído (UF) e fenol-formaldeído (FF) nas proporções de $5 \%$ e $8 \%$, com base na massa seca dos strands, sendo ensaiadas conforme ASTM D 1037 e comparadas com as normas CSA O437.0 e ANSI A.208.1. Os valores das propriedades mecânicas, exceto ligação interna, das chapas de cipreste usando a resina FF superaram as especificações das normas CSA O437.0 (classe O-2) e ANSI A.208.1, sendo de melhor desempenho a de $8 \%$ de adesivo. Em geral, as chapas apresentaram baixa estabilidade dimensional. $\mathrm{O}$ aumento no teor de resina levou ao melhor desempenho nas propriedades mecânicas e estabilidade dimensional. As chapas de seringueira apresentaram propriedades mecânicas acima do especificado pelas normas citadas, sendo que na ligação interna apenas aquelas coladas com 8\% de FF atendeu à norma CSA O437.0.

Palavras-chave: OSB; ureia-formaldeído; fenol-formaldeído; propriedades físicas e mecânicas.
\end{abstract}

\begin{abstract}
Oriented strandboard made of pine, cypress and rubberwood. This research evaluated the physicalmechanical properties of oriented strandboards (OSB) using strands of pine, cypress and a mixture of two clones, of rubberwood. The ratio of face to core layer was 1:2:1. Boards measuring $450 \times 450 \mathrm{x}$ $13 \mathrm{~mm}$ were bonded with $5 \%$ and $8 \%$ of urea-formaldehyde (UF) and phenol-formaldehyde (FF) resins, based on the dry weight of the particles, with density of $0.75 \mathrm{~g} / \mathrm{cm}^{3}$. The boards were evaluated according to ASTM D 1037 and compared with CSA O437.0 and ANSI A.208.1 standards. In general, as the resin level increased, mechanical and physical properties also improved, and panels with $8 \%$ FF resin showed the best performance. Cypress OSB made with FF resin showed higher mechanical properties, except internal bond than required by aforementioned standards. OSB panels were also dimensionally unstable since water absorption and thickness swelling values were very high. The mechanical properties of rubberwood OSB were higher than required by CSA O437.0 and ANSI A.208.1, except for internal bond, since only panels bonded with $8 \%$ of FF resin met the requirements of the CSA O437.0 standard.
\end{abstract}

Keywords: OSB; resin; urea- and phenol-formaldehyde; physical and mechanical properties.

\section{INTRODUÇÃO}

A silvicultura brasileira continua a se potencializar como vetor de desenvolvimento socioeconômico e ambiental do país. As crescentes restrições de caráter ambiental quanto ao uso de 
madeiras nativas têm aumentado a importância das madeiras de reflorestamento junto ao comércio internacional.

São raros os estudos sobre a madeira de cipreste no Brasil (PEREIRA; HIGA, 2003; OKINO et al., 2004a; 2005; 2006a; 2007), subestimando-se o potencial dessa espécie devido à falta de informações tecnológicas e principalmente silviculturais e impossibilitando o incentivo ao seu reflorestamento, ao contrário do que já vem ocorrendo em muitos países da América Latina, África e Oriente Médio. Na África existem florestas homogêneas públicas e privadas com as espécies Cupressus lusitânica e $C$. sempervirens. A Venezuela e a Colômbia dispõem de programas de melhoramento genético para melhor aproveitamento dessa espécie (LADRACH, 1993a; 1993b; VEBLEN, 1978). No Brasil, esse estudo está muito defasado.

A área total estimada de seringueiras plantadas em todo o mundo é superior a 9 milhões de hectares, e no Brasil foi de 103 mil hectares em 2004. Como a madeira apresenta alta suscetibilidade ao ataque de fungos e insetos, devido ao elevado teor de amido e açúcares, é imprescindível um tratamento profilático logo após o corte, em período inferior a 24 h (SBS, 2003a). A seringueira é importante porque, além do látex e da madeira, estima-se que árvores de 15 anos de idade retiram anualmente da atmosfera 16,6 toneladas de carbono por hectare, enquanto áreas com pastagens absorvem 14,3 toneladas de carbono por hectare (SBS, 2003b).

Yusoff; Horie (1997), citados por Mendes et al. (2002), estudando novas espécies para produção de OSB, comprovaram que a madeira de seringueira apresentou grande potencial para produção de painel estrutural, haja vista satisfazer os níveis aceitáveis das propriedades.

O Brasil não possui tradição quanto ao uso da madeira de seringueira, enquanto os países asiáticos a vêm usando para diversos fins, inclusive móveis e painéis reconstituídos, devido à sua cor clara, bela textura/desenho, facilidade em receber pigmentos, fácil processamento e boa qualidade em acabamento. A sua demanda tem aumentado na fabricação de chapas aglomeradas, de chapas de MDF e de chapas de cimento-madeira (THONG; NOR, 1993; KILLMANN; HONG, 2000).

A difusão quanto ao uso da madeira de seringueira em painéis reconstituídos tem sido feito por Okino et al. (2000; 2001; 2003), relatando os valores das propriedades mecânicas e de estabilidade dimensional.

A intensificação dos investimentos em florestas no Brasil, nos últimos cinco anos, veio modificar a distribuição etária dos plantios de Pinus, e hoje, do total de 1,8 milhão de hectares plantados, $26 \%$ possuem menos de cinco anos de idade. Atualmente, há cerca de 4,7 milhões de hectares com plantios de espécies de Pinus e Eucalyptus. Desse total, o eucalipto responde por cerca de $64 \%$ e o pinus por aproximadamente $36 \%$, sendo que a grande maioria é de Pinus taeda (SANT'ANNA et al., 2004; HOEFLICH, 2004).

O mercado de painéis OSB ainda está em fase de consolidação, no entanto, as perspectivas são animadoras, tendo em vista sua aplicação nas indústrias de construção civil, de embalagens e na indústria moveleira (IWAKIRI et al., 2003a).

Biblis (1985) confeccionou OSB de três camadas usando madeiras de folhosas e concluiu que a mistura de espécies com alta e baixa densidade era propícia para o uso na construção habitacional (house sheathing). Biblis (1989) também testou amostragens de OSB de três procedências diferentes de southern pine e constatou diferenças na rigidez e no MOE, na direção paralela. McNatt et al. (1992) concluíram que o alinhamento das partículas nas faces melhorava os módulos de elasticidade e de ruptura na mesma direção do alinhamento e que nem o alinhamento perpendicular do miolo, nem o alinhamento unidirecional melhoraram o desempenho do painel quando comparado com o alinhamento nas faces e miolo não orientado. A ligação interna e o inchamento em espessura não foram afetados pelo alinhamento das partículas.

Gouveia (2001), estudando o comportamento das espécies $E$. grandis e $P$. elliottii na produção de chapas de partículas orientadas, usando a resina fenólica a 4\%, $6 \%$ e $8 \%$, acrescida de $1 \%$ de emulsão de parafina, concluiu que as melhores chapas foram com $6 \%$ e $8 \%$ de adesivo. Nenhum tratamento teve êxito quanto ao inchamento em espessura e absorção de água, sendo satisfatório para o teste de expansão linear.

Mendes et al. (2000) usaram strands de $85 \mathrm{~mm}$ de comprimento de $P$. taeda na confecção de chapas OSB, de camada única e tripla, na proporção $1: 1: 1$, com $6 \%$ de resina $F F, 0,65 \mathrm{~g} / \mathrm{cm}^{3}$ de massa específica nominal e três larguras de strands. Os valores de MOE; MOR foram superiores no sentido 
perpendicular às fibras nos painéis de três camadas, mas no sentido paralelo esses valores foram superiores nos painéis de camada única. Mendes et al. (2002) estudaram o comportamento de pinus tropicais, como o P. taeda, o P. maximinoi, o P. tecunumanii, o $P$. caribaea var. hondurensis, o $P$. oocarpa e o $P$. chiapensis, e concluíram que as espécies produziram painéis de qualidade semelhante, atendendo às especificações mínimas da norma canadense CSA O437.0 (1993). Mendes et al. (2007) testaram tipos alternativos de resinas melamina-ureia-formaldeído e fenol-melamina-ureia-formaldeído na produção de chapas OSB com misturas de clones de Eucalyptus spp. Os valores de MOE e MOR foram afetados pelos tipos de resinas, enquanto que a ligação interna e a estabilidade dimensional não foram.

Iwakiri et al. (2003b) concluíram que a composição de chapa OSB de E. grandis com a relação 1:3:1, com $6 \%$ de resina fenol-formaldeído e densidade nominal de $0,7 \mathrm{~g} / \mathrm{cm}^{3}$, apresentou melhor balanço das propriedade de flexão estática entre os sentidos paralelo e perpendicular ao plano da chapa. Iwakiri et al. (2004), ao avaliar o potencial de utilização de E. grandis, E. saligna, E. dunnii, E. tereticornis, E. citriodora e E. maculata na produção de chapas OSB com densidade nominal de $0,70 \mathrm{~g} / \mathrm{cm}^{3}$ e $6 \%$ de resina $\mathrm{FF}$, demonstraram que o $E$. grandis apresentou propriedades físico-mecânicas similares ou superiores às das chapas de Pinus taeda.

Malanit; Laemsak (2007) estudaram o efeito da orientação nas propriedades físicas e mecânicas de chapas OSB de seringueira e concluíram que o alinhamento dos strands melhorou significativamente as propriedades de resistência e rigidez na direção do alinhamento, porém o efeito foi sutil em relação ao inchamento em espessura e ligação interna.

Este trabalho teve por objetivo avaliar as propriedades físicas e mecânicas de chapas de partículas orientadas de OSB, com strands de $200 \mathrm{~mm}$ de comprimento, usando as madeiras de pinus (Pinus taeda), de seringueira (Hevea brasiliensis) e de cipreste (Cupressus spp.), coladas com as resinas ureia-formaldeído e fenol-formaldeído.

\section{MATERIAL E MÉTODOS}

\section{Coleta do material}

Cipreste (Cupressus spp.) de Brasília.

Toras de aproximadamente 20 a 30 anos de idade de cipreste foram coletadas na região urbana

\section{Seringueira (Hevea brasiliensis Müll. Arg)}

Foram coletadas aleatoriamente 12 árvores de dois clones, Tjir 16 e RRIM 600, cultivados no estado de São Paulo, nos municípios de Tabapuã e Tabatinga, respectivamente. O seringal do clone Tjir 16, com aproximadamente 30 anos de idade, estava em fase de abate e o seringal do clone RRIM 600, com apenas 17 anos de idade, estava em plena atividade resinífera.

Pinus (Pinus taeda L.)

Pranchas de 18 anos de idade foram doadas pela empresa Placas do Paraná, sediada em Curitiba (PR).

\section{Preparação das partículas}

Os toretes e pranchões foram desdobrados em blocos de 50 x $150 \times 200 \mathrm{~mm}$ e transformados em partículas em um picador de disco, marca Bezner, de alta velocidade. A dimensão média das partículas de cipreste foi de $0,75 \times 33,7 \times 200,8 \mathrm{~mm}$, da seringueira de $0,80 \times 38,2 \times 200,0 \mathrm{~mm}$ e a de pinus de $0,74 \times$ 41,2 × 201,5 mm. As partículas foram classificadas em peneiras vibratórias, coletando-se aquelas que passaram na malha de abertura de $24 \mathrm{~mm}$ e ficaram retidas na de $8 \mathrm{~mm}$. O material foi seco em estufa ventilada a $103{ }^{\circ} \mathrm{C}$, obtendo-se um teor de umidade médio de $3 \%$.

\section{Tipos de resinas e caracterização}

Foram utilizadas as resinas ureia-formaldeído CR 8080 (UF), de baixa emissão de formol livre, e fenol-formaldeído CR 7010 (FF), ambas cedidas pela Schenectady Crios S.A./Rio Claro, SP. A resina de UF continha 64,0\% de sólidos resinosos, conforme a norma ASTM D 1490 - 67 (1972), e viscosidade média de $450 \mathrm{mPa} . \mathrm{s}(\mathrm{cP})$, medida em um aparelho Brookfield VR-110, a $25^{\circ} \mathrm{C}$. Na confecção de chapas 
com a resina UF, foi adicionado o endurecedor cloreto de amônio $\left(\mathrm{NH}_{4} \mathrm{Cl}\right)$ na proporção de $5 \%$ sobre a massa total do adesivo. A resina de FF continha $46,8 \%$ de sólidos resinosos, conforme a norma ASTM D 4426 - 84 (1984) e uma viscosidade média de $550 \mathrm{mPa} . \mathrm{s}$ (cP). Usou-se $5 \%$ e $8 \%$ de sólidos resinosos baseados na massa seca de partículas, sem adição de emulsão de parafina.

\section{Confecção da chapa}

A aplicação da resina foi feita em um misturador rotativo construída pelo LPF, com o material seco sendo impregnado com a resina durante 7 minutos. $O$ material resultante foi distribuído manualmente num formador de colchão e prensado em estágios. $\mathrm{O}$ ciclo de prensagem consistiu em aplicar uma pré-prensagem até que o centro do colchão atingisse $100{ }^{\circ} \mathrm{C}$, aliviando-se a pressão por 1 minuto e depois prensando por 5 minutos adicionais a $190{ }^{\circ} \mathrm{C}$ e $4,0 \mathrm{MPa}$ de pressão. Esse ciclo foi escolhido tendo em vista a ocorrência de bolhas (blow-up) durante a confecção das chapas de cipreste. Cada chapa foi composta de três camadas orientadas perpendicularmente entre si, numa proporção de face/miolo/face de 1:2:1, espessura de $13 \mathrm{~mm}$ e massa específica nominal de $0,75 \mathrm{~g} / \mathrm{cm}^{3}$.

Após a climatização, em condições controladas de umidade relativa de $65 \pm 2 \%$ e temperatura de $20 \pm 1{ }^{\circ} \mathrm{C}$, as chapas tiveram suas bordas aparadas para $430 \times 430 \mathrm{~mm}$. De cada chapa retiraram-se seis corpos-de-prova (duas amostras para cada uma das três repetições) para cada direção, para a execução dos ensaios de flexão estática (módulo de ruptura - MOR, módulo de elasticidade - MOE e tensão no limite proporcional - TLP), e doze corpos-de-prova (quatro amostras para cada uma das três repetições) para os demais testes físicos (absorção de água - AA, inchamento em espessura - IE, massa específica aparente M.E.A., teor de umidade - TU) e mecânicos (ligação interna - LI, Arranque de parafuso - AP e Dureza Janka - DJ), independentemente da orientação. Os corpos-de-prova foram testados de acordo com a norma ASTM D 1037 - 99 (2000), utilizando-se a máquina universal de testes Instron modelo 1127, e comparados com as normas CSA O437.0 (1993) e ANSI A.208.1 (1993).

\section{Delineamento experimental}

O experimento constou de um delineamento fatorial, onde a variável espécie de madeira foi analisada, por conveniência, em separado. O delineamento por espécie foi composto de dois tipos de resinas sintéticas (UF e FF), duas proporções de resina (5\% e 8\%) e três repetições, com 12 chapas por espécie e 36 no total do experimento.

Os resultados dos testes foram submetidos à Análise de Variância (ANOVA), utilizando-se o programa Statistical Package for Social Science (SPSS), e para a comparação entre os tratamentos usaram-se os testes de Tukey HSD em nível de 5\% de significância. Para os tratamentos das chapas OSB de pinus, foi usado o teste de Ryan-Einot-Gabriel-Welsch baseado no teste F (REGW-F). Como a ANOVA da propriedade de M.E.A. não detectou diferença significativa entre os tratamentos e o teste de Tukey HSD apresentou, foi escolhido o teste de REGW-F por ser mais robusto. Essa discrepância pode acontecer quando a significância do teste $\mathrm{F}$ fica na margem entre a aceitação ou não da hipótese de que as médias são iguais, como no caso do teste de M.E.A. $(p=0,051$ para $\alpha=0,05)$

\section{RESULTADOS E DISCUSSÕES}

\section{Propriedades mecânicas}

Os dados das propriedades mecânicas de chapas OSB de cipreste, seringueira e pinus estão listados na tabela 1.

Segundo a tabela 1, os valores de MOR na direção paralela de todas as chapas OSB superaram o mínimo especificado para a classe O-2 da norma canadense CSA O437.0, que é de 29,0 MPa, com exceção das chapas OSB de pinus com 5\% de resina UF e de cipreste com 5\% e 8\% dessa mesma resina. Os valores de MOR na direção perpendicular superaram o valor mínimo exigido para a classe O-2, que é de 12,4 MPa, com exceção da chapa de cipreste com 5\% de UF. Os valores absolutos de MOR, tanto no sentido paralelo quanto no perpendicular, foram mais elevados para as chapas OSB de seringueira usando a resina UF, porém com a resina $\mathrm{FF}$ as chapas de cipreste apresentaram melhor performance. A razão entre o valor médio de MOR paralelo/perpendicular variou de 1,0 a 1,5 para o pinus, 1,6 a 2,0 para a seringueira e de 1,1 a 1,8 para o cipreste. 
Tabela 1. Resultados das propriedades mecânicas de painéis de OSB feitos com partículas de Pinus taeda, Hevea brasiliensis e Cupressus spp.

Table 1. Values of average mechanical properties of OSB panels made of Pinus taeda, Hevea brasiliensis and Cupressus spp.

\begin{tabular}{|c|c|c|c|c|c|c|}
\hline \multirow{2}{*}{ Tratamento } & \multicolumn{2}{|c|}{ Pinus $^{7}$} & \multicolumn{2}{|c|}{ Seringueira $^{8}$} & \multicolumn{2}{|c|}{ Cipreste $^{8}$} \\
\hline & Paralelo & Perpendicular & Paralelo & Perpendicular & Paralelo & Perpendicular \\
\hline & \multicolumn{6}{|c|}{ Módulo de Ruptura $^{2}$ (MPa) } \\
\hline $\mathrm{UF}^{1}$ & $21,5^{\mathrm{A}}(7,6)$ & $17,2^{\mathbf{A}}(2,8)$ & $40,2^{\mathbf{A}}(11,0)$ & $24,9^{\mathrm{A}}(7,1)$ & $21,4^{\mathrm{A}}(6,1)$ & $11,8^{\mathrm{A}}(3,9)$ \\
\hline UF8 & $35,1^{\mathbf{A B}}(2,2)$ & $23,2^{\mathbf{A}}(3,9)$ & $39,5^{\mathrm{A}}(5,5)$ & $24,2^{\mathbf{A}}(2,9)$ & $24,8^{\mathrm{A}}(7,7)$ & $23,6^{\mathrm{AB}}(7,5)$ \\
\hline FF5 & $33,2^{\mathbf{A B}}(12,0)$ & $32,3^{\mathbf{A B}}(6,7)$ & $39,1^{\mathbf{A}}(7,6)$ & $19,9^{\mathbf{A}}(2,5)$ & $55,7^{\mathrm{B}}(7,5)$ & $37,0^{\mathrm{B}}(7,8)$ \\
\hline FF8 & $53,9^{\mathbf{B}}(12,6)$ & $41,2^{\mathbf{B}}(10,6)$ & $55,5^{\mathrm{A}}(12,6)$ & $30,4^{\mathbf{A}}(7,7)$ & $57,7^{\mathrm{B}}(2,7)$ & $35,2^{\mathrm{B}}(1,2)$ \\
\hline \multirow[t]{2}{*}{ Requisito $^{4}$} & \multicolumn{3}{|c|}{ Paralela (29,0 MPa) } & \multicolumn{3}{|c|}{ Perpendicular (12,4 MPa) } \\
\hline & \multicolumn{6}{|c|}{ Módulo de Elasticidade $^{2}(\mathrm{GPa})$} \\
\hline UF5 & $4,3^{\mathbf{A}}(1,2)$ & $1,7^{\mathbf{A}}(0,5)$ & $7,6^{\mathbf{A}}(1,3)$ & $2,9^{\mathrm{A}}(0,6)$ & $3,5^{\mathrm{A}}(1,6)$ & $1,3^{\mathrm{A}}(0,5)$ \\
\hline UF8 & $5,5^{\mathbf{A B}}(0,1)$ & $2,0^{\mathbf{A}}(0,3)$ & $7,6^{\mathbf{A}}(0,7)$ & $2,7^{\mathbf{A}}(0,5)$ & $4,1^{\mathrm{AB}}(1,1)$ & $2,9^{\mathrm{AB}}(1,3)$ \\
\hline FF5 & $7,0^{\mathrm{C}}(1,4)$ & $2,7^{\mathbf{A B}}(0,4)$ & $6,9^{\mathbf{A}}(0,5)$ & $2,0^{\mathbf{A}}(0,6)$ & $6,8^{\mathrm{B}}(0,8)$ & $3,6^{\mathrm{B}}(0,5)$ \\
\hline FF8 & $6,1^{\mathbf{A B}}(0,4)$ & $3,2^{\mathbf{B}}(0,5)$ & $8,3^{\mathbf{A}}(1,0)$ & $3,3^{\mathbf{A}}(0,9)$ & $6,2^{\mathrm{AB}}(0,6)$ & $3,6^{\mathrm{B}}(0,3)$ \\
\hline \multirow[t]{2}{*}{ Requisito $^{4}$} & \multicolumn{3}{|c|}{ Paralela (5,5 GPa) } & \multicolumn{3}{|c|}{ Perpendicular $(1,5 \mathrm{GPa})$} \\
\hline & \multicolumn{6}{|c|}{ Tensão no Limite Proporcional $^{2}(\mathrm{MPa})$} \\
\hline UF5 & $19,4^{\mathbf{A}}(5,6)$ & $14,3^{\mathbf{A}}(2,0)$ & $34,4^{\mathbf{A}}(8,4)$ & $20,0^{\mathrm{A}}(5,1)$ & $18,7^{\mathrm{A}}(6,0)$ & $10,0^{\mathrm{A}}(3,5)$ \\
\hline UF8 & $29,5^{\mathbf{A B}}(2,2)$ & $19,1^{\mathbf{A B}}(2,4)$ & $33,3^{\mathbf{A}}(5,0)$ & $19,6^{\mathbf{A}}(2,8)$ & $21,3^{\mathrm{A}}(7,0)$ & $20,3^{\mathrm{AB}}(6,2)$ \\
\hline FF5 & $29,2^{\mathbf{A B}}(10,6)$ & $25,7^{\mathbf{B C}}(5,3)$ & $32,3^{\mathbf{A}}(6,4)$ & $16,4^{\mathbf{A}}(2,7)$ & $47,0^{\mathrm{B}}(5,7)$ & $31,5^{\mathrm{B}}(6,9)$ \\
\hline FF8 & $44,5^{\mathbf{B}}(9,2)$ & $32,5^{\mathrm{C}}(6,9)$ & $47,1^{\mathbf{A}}(10,5)$ & $25,2^{\mathrm{A}}(6,6)$ & $49,1^{\mathrm{B}}(4,2)$ & $30,7^{\mathrm{B}}(0,6)$ \\
\hline \multirow[t]{2}{*}{ Tratamento } & \multicolumn{2}{|c|}{ Pinus $^{7}$} & \multicolumn{2}{|c|}{ Seringueira $^{8}$} & \multicolumn{2}{|c|}{ Cipreste $^{8}$} \\
\hline & \multicolumn{6}{|c|}{ Ligação Interna $^{3}(\mathrm{MPa})$} \\
\hline UF5 & \multicolumn{2}{|c|}{$0,06^{\mathrm{A}}(0,03)$} & \multicolumn{2}{|c|}{$0,15^{\mathrm{A}}(0,03)$} & \multicolumn{2}{|c|}{$0,04^{\mathrm{A}}(0,01)$} \\
\hline UF8 & \multicolumn{2}{|c|}{$0,09^{\mathbf{A B}}(0,01)$} & \multicolumn{2}{|c|}{$0,20^{\mathbf{A}}(0,05)$} & \multicolumn{2}{|c|}{$0,06^{\mathrm{A}}(0,02)$} \\
\hline FF5 & \multicolumn{2}{|c|}{$0,14^{\mathbf{A B}}(0,04)$} & \multicolumn{2}{|c|}{$0,17^{\mathbf{A}}(0,05)$} & \multicolumn{2}{|c|}{$0,23^{\mathrm{B}}(0,05)$} \\
\hline FF8 & 0,20 & $(0,08)$ & 0,41 & $(0,05)$ & 0,32 & $(0,07)$ \\
\hline Requisito $^{4}$ & & & $(0,345$ & MPa) & & \\
\hline & & & Arranque de & $\operatorname{arafuso}^{3}(\mathrm{~N})$ & & \\
\hline UF5 & 908 & $(131)$ & 1.976 & (219) & 1.083 & ${ }^{A}(228)$ \\
\hline UF8 & 1.338 & B $(170)$ & 2.010 & ${ }^{A}(89)$ & 1.378 & A (198) \\
\hline FF5 & 1.509 & ${ }^{3 C}(228)$ & 2.714 & (227) & 1.419 & A $(257)$ \\
\hline FF8 & 1.76 & $\mathrm{C}_{(52)}$ & 2.589 & (231) & 1.986 & B (151) \\
\hline Requisito $^{5}$ & & & (1.10 & $\mathrm{N})$ & & \\
\hline & & & Dureza J & $\mathrm{nka}^{3}(\mathrm{~N})$ & & \\
\hline UF5 & 5.530 & ${ }^{\mathrm{A}}(431)$ & 6.756 & $(503)$ & 5.993 & ${ }^{A}(296)$ \\
\hline UF8 & 6.794 & (1.044) & 5.837 & (469) & 6.459 & $\mathrm{~A}(541)$ \\
\hline FF5 & 6.64 & 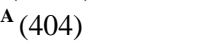 & 7.519 & (1.710) & 6.393 & A $(470)$ \\
\hline FF8 & 6.630 & ${ }^{A}(497)$ & 6.288 & ${ }^{A}(262)$ & 7.031 & ${ }^{A}(338)$ \\
\hline Requisito $^{6}$ & & & $(2.22$ & $5 \mathrm{~N})$ & & \\
\hline
\end{tabular}

Números entre parênteses são desvios-padrões. ${ }^{1}$ UF: ureia-formaldeído; FF: fenol-formaldeído, 5 ou 8: porcentagem de resina; ${ }^{2}$ : Média de 6 corpos-de-prova ( 2 para cada uma das 3 repetições) para cada orientação; ${ }^{3}$ : Média de 12 corpos-de-prova (4 para cada uma das 3 repetições, independentemente da orientação); ${ }^{4}$ : Valor mínimo exigido segundo requisito da norma canadense CSA O437.0, classe $\mathrm{O}-2$, nas direções paralela e perpendicular para painéis de OSB ${ }^{5}$ : Valor mínimo exigido segundo a norma ANSI A.208.1 para chapas de aglomerado de partículas; ${ }^{6}$ : Valor mínimo exigido pela norma CSA O437.0, classe R-1, para painéis waferboard $;^{7}$ : Valores de média, para cada propriedade, numa mesma coluna, seguidos da mesma letra não diferem estatisticamente entre si em nível de $5 \%$ de significância usando o teste de Ryan-Einot-Gabriel-Welsch F; ${ }^{8}$ : Valores de média, para cada propriedade, numa mesma coluna, seguidos da mesma letra não diferem estatisticamente entre si em nível de 5\% de significância usando o teste de Tukey HSD.

Os valores de MOE na direção paralela foram maiores do que o mínimo especificado para a classe O-2 da norma CSA O437.0, que é de 5,5 GPa, com exceção das chapas OSB de pinus com 5\% de resina UF e de cipreste com $5 \%$ e $8 \%$ dessa mesma resina. Os valores de MOE na direção perpendicular 
superaram o mínimo especificado para a classe O-2, que é de 1,5 GPa, com exceção das chapas OSB de cipreste com 5\% de resina UF. Os valores absolutos de MOE no sentido paralelo foram iguais ou superiores para as chapas OSB de seringueira. A razão entre o valor médio de MOE paralela/perpendicular para as chapas OSB variou de 1,9 a 2,8 vezes para o pinus, de 2,4 a 3,5 para a seringueira e de 1,4 a 2,7 vezes para o cipreste.

Os valores de TLP na direção paralela das chapas OSB confeccionadas variaram de 18,7 a 49,1 $\mathrm{MPa}$, e na perpendicular, de 10,0 a 32,5 MPa. A razão entre o valor médio de TLP paralela/perpendicular variou de 1,1 a 1,5 para as chapas OSB de pinus, de 1,7 a 2,0 para a seringueira e de 1,1 a 1,9 para as de cipreste. O valor de TLP é pouco explorado, apesar de poder ser usado como fator de segurança na previsão de ruptura, e não tem especificação em normas, como no caso do MOR e MOE.

Os valores de ligação interna variaram de 0,04 a $0,41 \mathrm{MPa}$. No geral, todos os valores de ligação interna não atenderam a norma canadense, com exceção das chapas OSB de seringueira com $8 \%$ de resina FF. Os baixos valores de ligação interna refletem uma irregular distribuição do adesivo e a nãouniformidade de deposição das partículas durante a formação do colchão, que podem ter sido afetadas pelo longo comprimento dos strands.

No geral, o aumento do teor de resina resultou em melhoria nas propriedades mecânicas das chapas produzidas, assim como houve a confirmação de que a resina fenólica é mais eficiente do que a resina ureica em qualquer proporção testada.

A madeira de pinus, apesar da baixa massa específica, possui madeira de reação e uma estrutura celular não uniforme, com variação da massa específica dentro dos anéis de crescimento, podendo resultar em perfil de densidade vertical, mesmo trabalhando-se nas condições ótimas de processo, o que influencia negativamente nas propriedades de resistência. Mendes et al. (2000), usando P. taeda com 6\% de resina FF na proporção 1:1:1 (face:miolo:face), obtiveram dados de flexão estática de $20 \%$ a $50 \%$ maiores que os deste estudo, com exceção do MOR na direção paralela. Em outro estudo, Mendes et al. (2003) obtiveram dados de MOR e MOE de chapas OSB de P. taeda com 6\% de resina FF inferiores aos deste estudo na direção paralela, e superiores na direção perpendicular. Okino et al. (2004b) confeccionaram chapas OSB de $P$. taeda com resinas UF e FF usando strands de $80 \mathrm{~mm}$ e obtiveram melhor performance, entretanto os valores de TLP foram maiores, assim como os valores de MOE para as chapas coladas com a resina fenólica. Isso demonstra que o comprimento da partícula afetou quase todas as propriedades físicas e mecânicas das chapas. Estudos de Iwakiri et al. (2004), com chapas OSB de $P$. taeda com 6\% de resina FF e proporção face:miolo:face de 1:3:1, apresentaram valores de MOR e MOE $20 \%$ superiores.

Todos os valores de arranque de parafuso, independente da orientação das partículas, superaram o valor mínimo exigido pela norma americana ANSI A.208.1, grau M-3, que é de $1.100 \mathrm{~N}$ para chapas aglomeradas de média densidade, com exceção da chapa OSB de pinus com 5\% de resina UF. A melhor performance foi apresentada pelos quatro tratamentos das chapas OSB de seringueira.

Os valores de dureza Janka superaram o mínimo requerido para a classe R-1 (2.226 N), segundo a norma CSA O437.0, para chapas do tipo waferboard, e não houve diferença significativa entre os tratamentos dentro de cada espécie.

De modo geral, as propriedades mecânicas das chapas OSB de seringueira foram superiores às de pinus e de cipreste, o que se deve provavelmente às propriedades intrínsecas da madeira.

Suzuki; Takeda, citados por Mendes et. al. (2002), afirmaram que as propriedades de flexão estática são altamente afetadas pela proporção entre as camadas e que essas propriedades tendem a se igualarem tanto no sentido paralelo quanto no perpendicular quando as camadas atingem a razão de 1:2:1. Apesar dessa afirmação, as chapas confeccionadas não demonstraram essa tendência.

Em se tratando da madeira de cipreste, foi adotado o programa de fechamento da prensa em estágios, com o intuito de eliminar a possível formação de bolhas. Entretanto, esse procedimento não eliminou completamente tal problema. Esse fato confirma o que foi ressaltado por Maloney (1977) e Cloutier (1998), que espécies com alto teor de extrativos são propensas a bolhas no final do ciclo de prensagem. Segundo relatório interno do LPF, o Cupressus spp. usado neste estudo contém 3,5\% de extrativo somente em álcool-tolueno. Vidaurre et al. (2004) também citam que foi necessário o tempo de prensagem de $14 \mathrm{~min}$., em razão da ocorrência de estouro em tempos menores em relação aos satisfatórios 8 min., para se confeccionar chapas OSB com duas espécies nativas da Mata Atlântica, como o guapuruvu (Schizolobium parahyba (Vell.) Blake) e o mulungu (Erythrina verna Vell.). 
As propriedades de resistência das chapas orientadas estão associadas às características da matéria-prima (variáveis de entrada), tais como nós, proporção de madeira juvenil e tensões durante o processamento. Há de se ressaltar que a madeira de cipreste foi proveniente de florestas sem qualquer trato silvicultural, estando sujeita a muitos defeitos, como nós e madeira de compressão, entre outros.

\section{Propriedades físicas}

Os valores das propriedades físicas das chapas OSB de cipreste, seringueira e de pinus estão listados na tabela 2.

Tabela 2. Resultados das propriedades físicas de painéis de OSB feitos com strands de Pinus taeda, Hevea brasiliensis e Cupressus spp.

Table 2. Values of average physical properties of OSB panels made of Pinus taeda, Hevea brasiliensis and Cupressus spp. strands.

\begin{tabular}{|c|c|c|c|c|c|c|}
\hline \multirow{2}{*}{ Tratamento } & \multicolumn{2}{|c|}{ Pinus $^{4}$} & \multicolumn{2}{|c|}{ Seringueira } & \multicolumn{2}{|c|}{ Cipreste } \\
\hline & $2 \mathrm{~h}$ & $24 \mathrm{~h}$ & $2 \mathrm{~h}$ & $24 \mathrm{~h}$ & $2 \mathrm{~h}$ & $24 \mathrm{~h}$ \\
\hline \multicolumn{7}{|c|}{ Inchamento em espessura $^{1}(\%)$} \\
\hline${ }^{2}$ UF5 & $47,9^{\mathrm{A}}(5,5)$ & - & $45,9^{\mathrm{C}}(0,3)$ & $53,5^{\mathrm{C}}(1,1)$ & $59,7^{\mathrm{C}}(0,5)$ & $66,2^{\mathrm{B}}(5,8)$ \\
\hline UF8 & - & - & $42,3^{\mathrm{C}}(3,8)$ & $51,7^{\mathrm{C}}(4,6)$ & $39,3^{\mathrm{B}}(4,7)$ & $55,7^{\mathrm{B}}(4,1)$ \\
\hline FF5 & $48,5^{\mathrm{A}}(8,5)$ & $60,5(8,8)$ & $27,2^{\mathrm{B}}(5,1)$ & $32,5^{\mathrm{B}}(5,1)$ & $15,8^{\mathrm{A}}(3,9)$ & $27,3^{\mathrm{A}}(5,7)$ \\
\hline FF8 & $35,6^{\mathrm{A}}(6,8)$ & $46,2(8,2)$ & $12,0^{\mathrm{A}}(0,7)$ & $17,4^{\mathrm{A}}(1,5)$ & $8,4^{\mathrm{A}}(1,8)$ & $16,8^{\mathrm{A}}(2,7)$ \\
\hline Requisito $^{3}$ & \multicolumn{6}{|c|}{ Após 24h: 15,0 } \\
\hline \multicolumn{7}{|c|}{ Absorção de água $^{1}(\%)$} \\
\hline UF5 & $104,7^{C}(1,5)$ & $122,5^{C}(0,5)$ & $61,1^{\mathrm{C}}(4,7)$ & $75,8^{\mathrm{C}}(5,0)$ & $68,9^{\mathrm{D}}(1,5)$ & $87,9^{\mathrm{D}}(1,4)$ \\
\hline UF8 & $88,1^{\mathrm{B}}(3,4)$ & $109,9^{\mathrm{BC}}(1,0)$ & $57,3^{\mathrm{C}}(3,9)$ & $70,9^{\mathrm{C}}(2,4)$ & $51,6^{\mathrm{C}}(2,5)$ & $70,3^{\mathrm{C}}(2,0)$ \\
\hline FF5 & $80,4^{\mathrm{B}}(10,0)$ & $99,8^{\mathrm{AB}}(10,8)$ & $42,8^{\mathrm{B}}(4,0)$ & $58,7^{\mathrm{B}}(1,7)$ & $27,8^{\mathrm{B}}(1,2)$ & $50,4^{\mathrm{B}}(0,7)$ \\
\hline FF8 & $66,8^{\mathrm{A}}(9,5)$ & $86,9^{\mathrm{A}}(7,4)$ & $25,9^{\mathrm{A}}(2,8)$ & $44,5^{\mathrm{A}}(3,1)$ & $18,6^{\mathrm{A}}(3,4)$ & $42,4^{\mathrm{A}}(4,8)$ \\
\hline \multicolumn{7}{|c|}{ Massa específica aparente ${ }^{1}\left(\mathrm{~g} / \mathrm{cm}^{3}\right)$} \\
\hline UF5 & \multicolumn{2}{|c|}{$0,74^{\mathrm{A}}(0,01)$} & \multicolumn{2}{|c|}{$0,72^{\mathrm{A}}(0,05)$} & \multicolumn{2}{|c|}{$0,74^{\mathrm{A}}(0,02)$} \\
\hline UF8 & \multicolumn{2}{|c|}{$0,78^{\mathrm{A}}(0,02)$} & \multicolumn{2}{|c|}{$0,73^{\mathrm{A}}(0,02)$} & \multicolumn{2}{|c|}{$0,74^{\mathrm{A}}(0,02)$} \\
\hline FF5 & \multicolumn{2}{|c|}{$0,78^{\mathrm{A}}(0,02)$} & \multicolumn{2}{|c|}{$0,73^{\mathrm{A}}(0,03)$} & \multicolumn{2}{|c|}{$0,77^{\mathrm{A}}(0,02)$} \\
\hline FF8 & \multicolumn{2}{|c|}{$0,80^{\mathrm{A}}(0,03)$} & \multicolumn{2}{|c|}{$0,72^{\mathrm{A}}(0,03)$} & \multicolumn{2}{|c|}{$0,78^{\mathrm{A}}(0,02)$} \\
\hline \multicolumn{7}{|c|}{ Teor de umidade $(\%)$} \\
\hline UF5 & \multicolumn{2}{|c|}{$9,19^{\mathrm{AB}}(0,12)$} & \multicolumn{2}{|c|}{$8,00^{\mathrm{A}}(0,38)$} & \multicolumn{2}{|c|}{$8,01^{\mathrm{B}}(0,17)$} \\
\hline UF8 & \multicolumn{2}{|c|}{$8,97^{\mathrm{A}}(0,34)$} & \multicolumn{2}{|c|}{$7,99^{\mathrm{A}}(0,08)$} & \multicolumn{2}{|c|}{$7,75^{\mathrm{AB}}(0,06)$} \\
\hline FF5 & \multicolumn{2}{|c|}{$9,15^{\mathrm{A}}(0,07)$} & \multicolumn{2}{|c|}{$8,35^{\mathrm{A}}(0,30)$} & \multicolumn{2}{|c|}{$7,68^{\mathrm{A}}(0,06)$} \\
\hline FF8 & \multicolumn{2}{|c|}{$9,59^{\mathrm{B}}(0,13)$} & \multicolumn{2}{|c|}{$9,08^{\mathrm{B}}(0,48)$} & \multicolumn{2}{|c|}{$8,55^{\mathrm{C}}(0,08)$} \\
\hline
\end{tabular}

Valores de média, para cada propriedade, numa mesma coluna, seguidos da mesma letra não diferem estatisticamente entre si ao nível de 5\% usando o teste de Tukey HSD. Números entre parênteses são desvios padrões. ${ }^{1}$ : Média de 12 corpos-de-prova (4 para cada uma das 3 repetições), independentemente da orientação; ${ }^{2}:$ UF $=$ ureia-formaldeído, $F F=$ fenol-formaldeído, 5 ou $8=$ porcentagem de resina; ${ }^{3}$ : Valor máximo aceitável segundo requerimentos da norma canadense CSA O437.0, classe O-2, para painéis de espessura superior a $12,7 \mathrm{~mm} ;{ }^{4}:$ Valores de média, para cada propriedade, numa mesma coluna, seguidos da mesma letra não diferem estatisticamente entre si em nível de 5\% de significância usando o teste de Ryan-Einot-Gabriel-Welsch F.

As chapas OSB de seringueira e de cipreste com $8 \%$ de resina FF foram as que ficaram mais próximas dos requisitos de inchamento em espessura da norma CSA O437.0, classe O-2, que é de 15,0\%, após $24 \mathrm{~h}$ de imersão em água. As demais chapas ultrapassaram em muito esse limite, fato esse usualmente descrito na literatura. No caso das chapas OSB de pinus, não foi possível apresentar todos os resultados, uma vez os corpos-de-prova com a resina UF tiveram alguns strands descolados no processo de imersão, impossibilitando a medição da espessura final. As chapas de seringueira e de cipreste com a resina fenólica apresentaram os melhores resultados de inchamento em espessura após $2 \mathrm{~h}$, fato esse confirmado pelas características desse tipo de resina termorrígida. Os valores de inchamento em espessura após $24 \mathrm{~h}$ apresentaram a mesma tendência que após $2 \mathrm{~h}$.

Os comentários descritos para ligação interna também são válidos para o inchamento em espessura e a absorção de água, devido à característica não plana dos strands, os quais aumentam os espaços vazios interpartículas e, consequentemente, a absorção de água. Segundo Winistofer e Xu (1996), 
há dois tipos de água envolvidos no teste de absorção de água: a água de ligação (entre a parede celular) e a água livre (dentro do lume da célula). Acredita-se que a água de ligação seja a responsável pela expansão na espessura, chegando ao máximo de $25 \%$ quando a parede celular está saturada. O aumento contínuo após 8 e 12 horas de imersão em água pode ser atribuído principalmente ao ganho em água livre. Esse ganho poderia ter sido reduzido, caso se fizessem as medições antes de se efetuarem as pesagens dos corpos-de-prova.

Chapas mais densas geralmente tendem a sofrer maior inchamento em espessura, devido à maior quantidade de partículas e consequentemente à maior área superficial, além de maior número de sítios ativos $(\mathrm{OH})$ para a formação de pontes de hidrogênio. Ocorre também a liberação de tensões de prensagem durante a imersão dos corpos-de-prova, efeito conhecido como springback. Esse efeito é irrecuperável, ou plástico, e se converte na liberação parcial de deformação dos strands que ocorreu durante a prensagem a quente da chapa.

Segundo Wang; Winistofer (2000), mais de dois terços do inchamento em espessura ocorrem principalmente nas regiões de alta massa específica superficial durante os primeiros estágios de imersão em água. Segundo eles, durante a prensagem há um pré-aquecimento da superfície inferior do colchão de partículas em relação à superior, influenciando na consolidação do painel e acarretando a pré-cura da resina antes mesmo que seja atingida a espessura final. Em casos extremos, quando a razão de fechamento for de 40 a 60 segundos, há uma "delaminação" dos strands após imersão em água. Esse fato foi constatado ao se realizar o ensaio de absorção de água em corpos-de-prova da chapa OSB de pinus.

As porcentagens de absorção de água das chapas OSB após $2 \mathrm{~h}$ foram elevadas, sendo que as chapas OSB de seringueira e de cipreste apresentaram valores similares. Nessa classificação, nota-se que os melhores tratamentos foram para as chapas OSB de seringueira e cipreste usando a resina fenólica, e o pior tratamento foi para as chapas OSB de pinus, independentemente do tipo de resina aplicada. A absorção de água, após $24 \mathrm{~h}$, também foi elevada, apresentando a mesma tendência que após $2 \mathrm{~h}$.

Segundo Mendes et al. (2002), a incorporação da parafina ao colchão causa redução da higroscopicidade das partículas, pois esta reage quimicamente com a madeira, ocupando os sítios hidroxílicos higroscópicos e melhorando a estabilidade dimensional das chapas. Vale ressaltar que nesse estudo não foi adicionada parafina na confecção dos painéis de OSB.

Os resultados de M.E.A. variaram de 0,72 a $0,80 \mathrm{~g} / \mathrm{cm}^{3}$ e ficaram acima do inicialmente pretendido, talvez devido à distribuição irregular de material durante a formação manual do colchão. Não houve diferença significativa entre as M.E.A. dos tratamentos testados, sendo que as chapas OSB de seringueira foram as que mais se aproximaram da M.E.A. nominal. Cloutier (1998) afirma que, no Canadá, as indústrias produzem chapas OSB com massa específica na faixa de 0,63 a $0,67 \mathrm{~g} / \mathrm{cm}^{3}$, estando os painéis aqui confeccionados acima dessa faixa considerada ideal.

No geral, o teor de umidade médio das chapas confeccionadas variou de 7,68\% a 9,59\%.

O comprimento dos strands de seringueira foi uma variável crítica no processo de confecção das chapas, uma vez que apresentaram encaracolamento e textura áspera, não recebendo resina suficiente na parte interna, o que resultou em enfraquecimento da linha de cola, como citado por Wu e Piao (1999). Esse fenômeno provocou uma redução da ligação interna, pelo fato de uma menor área superficial de colagem estar em contato, além da baixa compressibilidade dos strands na zona superficial das chapas, provocando também baixa estabilidade dimensional.

Segundo Iwakiri (2005), as dimensões convencionais de partículas tipo strands usadas na produção de chapas orientadas estão em torno de $25 \mathrm{~mm}$ de largura, 90 a $150 \mathrm{~mm}$ de comprimento e 0,50 a $0,75 \mathrm{~mm}$ de espessura. Barnes (2000) afirma que a razão de esbeltez, que é a relação ótima entre o comprimento da partícula e a espessura, deve ser de 300. Por outro lado, Brumbaugh (1960) cita o intervalo de 150 a 250.

No caso das chapas OSB de pinus, a razão de compactação média foi de 1,92. A altura inicial do colchão foi de $100 \mathrm{~mm}$ e a espessura final da chapa orientada foi de $13 \mathrm{~mm}$, resultando em uma redução de 8,0 vezes na espessura. O índice de esbeltez médio foi igual a 272, estando em concordância com o limite entre 150 e 300, citado por Shuler e Kelly (1976) e Barnes (2000).

No caso das chapas OSB de seringueira, a razão de compactação média foi de 1,10 , não atingindo o mínimo de 1,3 citado por Iwakiri (2005). Esse valor indica que a orientação ficou prejudicada em razão do encaracolamento das partículas. O índice de esbeltez foi de 268 , estando em conformidade com os valores citados pela literatura.

A altura do colchão de partículas de cipreste foi de aproximadamente $100 \mathrm{~mm}$ e, ao final, a chapa ficou com $13 \mathrm{~mm}$ de espessura, ocorrendo uma redução de 7,7 vezes. A razão de compactação 
média foi de 1,47, superando o valor mínimo de 1,3 para que ocorra a densificação necessária à formação do painel (IWAKIRI, 2005). O valor do índice de esbeltez médio foi de 268, estando no intervalo citado por Shuler e Kelly (1976) e Barnes (2000).

\section{Efeito do tipo de resina}

Tomando como base o efeito do tipo de resina isoladamente, houve interação significativa de todas as propriedades das chapas OSB de cipreste e de pinus, exceto a dureza Janka. Quanto a chapas de seringueira, o tipo de resina interfere na estabilidade dimensional (IE e AA) e nas propriedades mecânicas de arranque de parafuso e de ligação interna. Estatisticamente, percebe-se que o tipo de resina é uma variável de extrema importância, tanto do ponto de vista econômico, diretamente relacionado à produção de painéis reconstituídos, como do técnico, associado à qualidade e usos do produto final.

\section{Efeito do teor de resina}

Analisando a quantidade de resina em separado, observa-se que foi estatisticamente significativa para as propriedades de estabilidade dimensional para as espécies de madeira estudadas. Quanto às propriedades mecânicas, as interações são limitadas à ligação interna, arranque de parafusos e dureza Janka. Quanto à flexão estática, somente o MOR paralelo de chapas de pinus sofreu influência do teor de resina.

\section{Efeito da interação entre as variáveis}

Quando se combinam os efeitos do tipo com a porcentagem de resina, observa-se que a interação é estatisticamente significativa somente para as propriedades de inchamento e absorção de água e o teor de umidade. A única propriedade mecânica que sofreu influência combinada dessas duas variáveis foi a ligação interna de chapas de OSB de seringueira, ou seja, essas duas variáveis podem ser tratadas individualmente, não havendo interferências entre si.

\section{Efeito das espécies}

Analisando o efeito das espécies sobre as propriedades das chapas produzidas, chegou-se à constatação de que a espécie de madeira não apresentou diferença significativa em nível de $5 \%$ de significância para o MOR e o MOE na direção perpendicular, o MOR na paralela, a dureza Janka e o inchamento em espessura, apresentando apenas um grupo homogêneo pelo teste de Tukey. Por outro lado, a espécie influenciou significativamente o MOE na paralela, a ligação interna, o arranque de parafuso e a absorção de água, apresentando apenas dois grupos homogêneos. Em relação a essas propriedades mecânicas, as chapas OSB de seringueira apresentaram-se como um grupo à parte, e com relação à estabilidade dimensional, as chapas OSB de cipreste foram mais estáveis em tempo menor, enquanto que as de seringueira foram mais estáveis em tempo maior.

As três madeiras atenderam ao mínimo exigido pela norma canadense ao usar a resina $\mathrm{FF}$, com exceção da ligação interna e inchamento em espessura, após $24 \mathrm{~h}$.

\section{CONCLUSÕES}

- As chapas de OSB de pinus com 5\% de resina UF não atenderam à norma canadense para o grau O2 , enquanto que a maioria dos tratamentos atingiu os valores mínimos exigidos.

- Todas as propriedades mecânicas das chapas OSB de seringueira e de cipreste foram superiores ao valor mínimo exigido pela norma CSA O437, classe O-2, com exceção da ligação interna.

- A madeira de cipreste, uma conífera exótica, necessita de estudos mais aprofundados para se tornar tecnicamente viável como matéria-prima à confecção de chapas OSB.

- A mistura dos clones RRIM 600 e Tjir 16 demonstrou-se tecnicamente viável como matéria-prima para a confecção de chapas OSB.

- Atribuiu-se a não conformidade dos valores de ligação interna e inchamento em espessura das chapas OSB com os requisitos das normas à não adição de parafina e à deficiência na distribuição da resina. No entanto, a redução da massa específica aparente das chapas ou o tratamento térmico superficial são considerações a serem estudadas.

- No geral, o efeito da quantidade de resina foi estatisticamente significativo para a estabilidade dimensional das chapas, e o seu incremento melhorou os valores das propriedades físicas e mecânicas. 
- O efeito do tipo de resina foi estatisticamente significativo para todas as propriedades estudadas de chapas OSB de pinus e de cipreste, exceto para a dureza Janka.

- O efeito da interação entre o tipo de resina e o teor foi estatisticamente significativo para as propriedades de AA, IE e teor de umidade.

- A análise do efeito da espécie mostrou que, no geral, chapas OSB de seringueira apresentaram melhores resultados para algumas propriedades, entre as madeiras estudadas.

- Novos estudos devem focar na otimização dos parâmetros do processo de confecção das chapas OSB com strands de $200 \mathrm{~mm}$.

\section{AGRADECIMENTOS}

O Laboratório de Produtos Florestais (LPF) agradece ao Dr. Marcelo Branco, da Schenectady CRIOS S.A., Rio Claro, SP, pela doação das resinas sintéticas, e também ao Sr. Roberto Trevisam, da Placas do Paraná S.A., Curitiba, PR, pelo material usado na confecção das chapas de OSB. A equipe agradece também aos técnicos João E. Anacleto, Sérgio Martinez, Getúlio F. de Almeida, Luiz D. Santana, Francisco L. de Araújo, Fernando A. de Oliveira, Ricardo P. de O. Santos e em especial ao Sr. Antônio de P. F. Lima, pela construção do misturador de partículas. Os autores estão em débito com a Sra. Theodora R. Fischli, pela pronta revisão ortográfica do artigo.

\section{REFERÊNCIAS}

AMERICAN NATIONAL STANDARD. ANSI A208.1: mat-formed wood particleboard: specification Gaithersburg: 1993. 9 p.

AMERICAN SOCIETY FOR TESTING AND MATERIALS. ASTM D 1037 - 99: standard test methods for evaluating properties of wood-base fiber and particle panel materials: Philadelphia, 2000. v. 04.09. (Annual Book of ASTM Standards).

AMERICAN SOCIETY FOR TESTING AND MATERIALS. ASTM D 1490 - 67: standard test method for nonvolatile content of urea-formaldehyde resin solutions: Philadelphia, 1972. v. 04.09. (Annual Book of ASTM Standards).

AMERICAN SOCIETY FOR TESTING AND MATERIALS. ASTM D 4426 - 84. standard test method for determination of percent nonvolatile content of liquid phenolic resins used for wood laminating: Philadelphia, 1984. v. 04.09. (Annual Book of ASTM Standards).

BARNES, D. An integrated model of the effect of processing parameters on the strength properties of oriented strand wood products. Forest Products Journal, Madison, v. 50, n. 11/12, p. 33-42, 2000.

BIBLIS, E. J. Properties of three-layer oriented strandboard from southern hardwoods. Forest Products Journal, Madison, v. 35, n. 2, p. 28-32, 1985.

BIBLIS, E. J. Properties of commercial OSB from three southern pine mills. Forest Products Journal, Madison, v. 39, n. 1, p. 55-57, 1989.

BRUMBAUGH, J. Effect of flake dimensions on properties of particle boards. Forest Products Journal, Madison, p. 243-246, 1960.

CANADIAN STANDARDS ASSOCIATION. Oriented strandboard and waferboard. Technical Bulletin SBA, Standard CSA O437.0-93. WILLOWDALE, Ontario, 18 p, 1993.

CLOUTIER, A. Oriented strandboard (OSB): raw material, manufacturing process, properties of woodbase fiber and particle materials. In: INTERNATIONAL SEMINAR ON SOLID WOOD PRODUCTS OF HIGH TECHNOLOGY, 1., Anais... Belo Horizonte: SIF/UFV/DEF, 1998. p. 173-185.

GOUVEIA, F. N. Produção de Chapas de partículas orientadas (OSB) a partir das espécies Eucalyptus grandis W. Hill ex Maiden e Pinus elliottii Engelm. 68 p. Dissertação (Mestrado em Ciência Florestal) - Universidade Federal de Viçosa, Viçosa, MG, 2001.

HOEFLICH, V. A. O papel das florestas para o desenvolvimento da sociedade brasileira. Revista da Madeira, edição especial, Curitiba, p. 14-20, ago. 2004. 
IWAKIRI, S.; MONTEFUSCO, A. R. G.; ZABLONSKY, K. M.; SIQUEIRA, K. P.; SALDANHA, L. K.; SOUZA, M. A. M. Produção de chapas de partículas "strand" com inclusão laminar - "Com-ply". Floresta e Ambiente, Seropédica, v. 10, n. 2, p. 30-35, 2003a.

IWAKIRI, S.; MENDES, L. M.; SALDANHA, L. K. Produção de chapas de partículas orientadas "OSB” de Eucalyptus grandis com diferentes teores de resina, parafina e composição em camadas. Ciência Florestal , Santa Maria, v. 13, n. 1, p. 89-94, 2003b.

IWAKIRI, S.; MENDES, L. M.; SALDANHA, L. K.; SANTOS, J. C. Utilização da madeira de eucalipto na produção de chapas de partículas orientadas - OSB. Cerne, Lavras, v. 10, n. 1, p. 46-52, 2004.

IWAKIRI, S. (Ed.). Painéis de madeira reconstituída. Curitiba: FUPEF, 2005. 247 p.

KILLMANN, W.; HONG, L. T. Rubberwood - the success of an agricultural by-product. Unasylva, Rome, v. 51, n. 2, p. 66-72, 2000.

LADRACH, W. E. Variação do crescimento de diversas procedências de ciprestes após dois e três anos de crescimento. Silvicultura, São Paulo, v. 8, n. 30, p. 340-342, 1993 a.

LADRACH, W. E. Ganhos genéticos com Cupressus lusitanica, ao longo de seis anos de melhoramento genético, na Colômbia. Silvicultura, São Paulo, v. 8, n. 30, p. 342-346, 1993 b.

MALANIT, P.; LAEMSAK, N. Effect of strand orientation on physical and mechanical properties of rubberwood oriented strandboard. Walailak Journal of Science \& Technology, Walailak, v. 4, n. 2, p. 215-223, 2007.

MALONEY, T. M. Modern particleboard and dry-process fiber board manufacturing. San Francisco, M. Freeman, 1977. 672 p.

McNATT, J. D.; BACH, L.; WELLWOOD, R. W. Contribution of flake alignment to performance of strandboard. Forest Product Journal, Madison, v. 42, n. 3, p. 45-50, 1992.

MENDES, L. M.; IWAKIRI, S.; MATOS, J. L.; KEINERT Jr., S.; SALDANHA, L. K. Avaliação do sistema de orientação de partículas na produção de painéis OSB (Oriented Strand Board). Cerne, Lavras, v. 6, n. 1, p. 001-008, 2000.

MENDES, L. M.; IWAKIRI, S.; MATOS, J. L. M.; KEINERT Jr, S.; SALDANHA, L. S. Pinus spp. na produção de painéis de partículas orientadas (OSB). Ciência Florestal, Santa Maria, v. 12, n. 2, p. 135145, 2002.

MENDES, L. M.; IWAKIRI, S.; MATOS, J. L. M.; KEINERT Jr., S.; SALDANHA, L. K. Efeitos da densidade, composição dos painéis e teor de resina nas propriedades de painéis OSB. Floresta e Ambiente, Seropédica, v. 10, n. 1, p. 01-17, 2003.

MENDES, S. A.; MENDES, L. M.; CHAVES, M. D.; MORI, F. A.; SILVA, J. R. M.; TRUGILHO, P. F. Utilização de resinas alternativas na produção de painéis OSB de clones de Eucalyptus spp. Cerne, Lavras, v. 13, n. 13, p. 257-263, 2007.

OKINO, E. Y. A.; SANTANA, M. A. E.; SOUZA, M. R. de; SOUSA, M. E. de. Ưtilização da madeira de Hevea brasiliensis Muell. Arg. na fabricação de chapas aglomeradas. Revista Árvore, Viçosa, MG, v. 24, n. 3, p. 431-347, 2000.

OKINO, E. Y. A.; SANTANA, M. A. E.; SOUZA, M. R.; SOUSA, M. E. de. Dimensional stability and mechanical properties of acetylated Hevea brasiliensis flakeboards. Journal of Tropical Forest Products, Kuala Lumpur, v. 7, n. 1, p. 96-102, 2001.

OKINO, E. Y. A.; SANTANA, M. A. E.; SOUZA, M. R.; SOUSA, M. E. de; ALVES, M. V. S. Propriedades físicas, mecânicas e durabilidade natural de chapas aglomeradas de Hevea brasiliensis Müll. Arg. In: CONGRESSO FLORESTAL BRASILEIRO, 8., 2003, São Paulo - SP. Anais do... São Paulo: SBS, 2003.

OKINO, E. Y. A.; SOUZA, M. R. de; SANTANA, M. A. E.; ALVES, M. V. da; SOUSA, M. E. de; TEIXEIRA, D. E. Evaluation of the physical and biological properties of particleboard and flakeboard made from Cupressus spp. International Biodeterioration \& Biodegradation, England, v. 53, p. 1-5, 2004a. 
OKINO, E. Y. A.; TEIXEIRA, D. E.; SOUZA, M. R. de; SANTANA, M. A. E.; SOUSA, M. E. de. Properties of oriented strandboard made of wood species from Brazilian planted Forest: Part 1: $80 \mathrm{~mm}-$ long strands of Pinus taeda L. Holz Roh Werkst, Berlim, v. 62, p. 221-224, 2004b.

OKINO, E. Y. A.; SOUZA, M. R. de; SANTANA, M. A. E.; ALVES, M. V. da; SOUSA, M. E. de; TEIXEIRA, D. E. Physico-mechanical properties and decay resistance of Cupressus spp. Cement-bonded particleboards. Cement \& Concrete Composites, England, v. 27, p. 333-338, 2005.

OKINO, E. Y. A.; CAMARGOS, J. A. A.; SANTANA, M. A. E.; MARQUES, M. H. B.; MARTINS, V. A.; SOUSA, M. E.; TEIXEIRA, D. E. Descrição dos caracteres tecnológicos da madeira de Cupressus glauca Lam. Scientia Forestalis, Piracicaba, n. 72, p. 39-48, 2006a.

OKINO, E. Y. A.; TEIXEIRA, D. E.; SANTANA, M. A. E.; SOUZA, M. R.; SOUSA, M. E. de. Propriedades de chapas de OSB feitas com espécies de madeiras de florestas plantadas no Brasil: Parte 1: strands de $80 \mathrm{~mm}$ de Hevea brasiliensis. In: CONGRESSO BRASILEIRO DE INDUSTRIALIZAÇÃO DA MADEIRA E DE PRODUTOS DE BASE FLORESTAL, 2., 2006, Anais... Curitiba: [s.n.], , 2006b. p. 1-8.

OKINO, E. Y. A.; TEIXEIRA, D. E.; DEL MENEZZI, C. H. S. Post-thermal treatment of oriented strandboard (OSB) made from cypress (Cupressus glauca Lam.). Maderas. Ciencia y Tecnología, Santiago, v. 9, n. 3, p. 199-210, 2007.

OKINO, E. Y. A.; ALVES, M. V. S.; TEIXEIRA, D. E.; SOUZA, M. R.; SANTANA, M. A. E. Biodegradação de chapas de partículas orientadas de pinus, eucalipto e cipreste expostas a quatro fungos apodrecedores. Scientia Forestalis, Piracicaba, n. 74, p. 67-74, 2007.

PEREIRA, J. C. D.; HIGA, R. C. V. Propriedades da madeira de Cupressus lusitanica Mill.. Colombo: EMBRAPA CNPF, 2003. 4 p. (Comunicado Técnico, n. 107).

SANT'ANNA Jr, M.; RAYZEL, T.; WANZUITA, M. M. C. Mercado internacional de produtos de madeira sólida de pinus. Revista da Madeira, edição especial, Curitiba, p. 6-8, ago. 2004.

SBS. 2003a. Dia a dia. Disponível em: <http://www.sbs.org.br >. Acesso em: 16/04/2003.

SBS. 2003b. Dia a dia. Disponível em:<http://www.sbs.org.br>. Acesso em: 10/11/2003.

SHULER, C. E.; KELLY, R. A. Effect of flake geometry on mechanical properties of Eastern Spruce flake-type particleboard. Forest Product Journal, Madison, v. 26, n. 6, p. 24-28, 1976.

THONG, H. L.; NOR, D. S. M. Present and potential uses of rubberwood - opportunities and future direction. In: INTERNATIONAL FORUM ON INVESTMENT OPPORTUNITIES IN THE RUBBERWOOD INDUSTRY, Kuala Lumpur, 1993.

VEBLEN, T. T. Guatemalan conifers. Unasylva, Rome, v. 29, n. 118, p. 25-30, 1978.

VIDAURRE, G. B.; SILVA, A. N.; ROCHA, J. D. S.; BRITO, E. O. Produção de chapas de partículas de madeira de duas espécies nativas da Mata Atlântica e suas combinações. Ciência Florestal, Santa Maria, v. 14, n. 1, p. 235-242, 2004.

WANG, S.; WINISTOFER, P. M. The effect of species and species distribution on the layer characteristic of OSB. Forest Products Journal, Madison, v. 50, n. 4, p. 37-44, 2000.

WINISTOFER, P. M.; XU, W. Layer water absorption of medium density fiberboard and oriented strandboard. Forest Products Journal, Madison, v. 46, n. 6, p. 69-72, 1996.

WU, Q.; PIAO, C. Thickness swelling and its relationship to internal bond strength loss of commercial oriented strandboard. Forest Products Journal, Madison, v. 49, n. 7/8, p. 50-55, 1999. 